

А. А. Андрианов, В. А. Андрианов, П. Джаккони, Р. Солдати, Индуцированная гравитация и образование Вселенной на доменной стенке в пятимерном пространстве-времени, ТМФ, 2006, том 148, номер 1, 422

DOI: https://doi.org/10.4213/tmf2054

Использование Общероссийского математического портала Math-Net.Ru подразумевает, что вы прочитали и согласны с пользовательским соглашением http://www . mathnet.ru/rus/agreement

Параметры загрузки:

IP : 107.22 .136 .117

26 апреля 2023 г., 14:00:24 


\title{
ИНДУЦИРОВАННАЯ ГРАВИТАЦИЯ И ОБРАЗОВАНИЕ ВСЕЛЕННОЙ НА ДОМЕННОЙ СТЕНКЕ В ПЯТИМЕРНОМ ПРОСТРАНСТВЕ-ВРЕМЕНИ
}

\begin{abstract}
Представлена модель в пятимерном пространстве-времени с сильным самодействием фермионов и индуцированным гравитационным взаимодействием. В режиме сильной связи в этой модели возникает спонтанное нарушение трансляционной симметрии, которое приводит к локализации легких частиц на $(3+1)$ мерной доме́нной стенке, вложенной в пятимерное пространство-время антиде Ситтера. Получено соответствующее низкоэнергетическое эффективное действие и исследованы вакуумные решения типа кинка в квазиплоской римановой метрике. Обсуждается $(3+1)$-мерная физика легких частиц и установлены фундаментальные соотношения между индуцированной гравитационной константой и кривизной в пятимерном пространстве-времени анти-де Ситтера, а также гравитационной ньютоновской константой нашей Вселенной.
\end{abstract}

Ключевые слова: индуцированная гравитация, динамическое нарушение симметрий, доме́нные стенки в пятимерном пространстве-времени.

Посвящается Ю. В. Новожилову к его 80-летию

\section{1. ВВЕДЕНИЕ}

Идея гравитации, полностью индуцированной полями материи в $(3+1)$-мерном пространстве-времени, была предложена в 60-е годы прошлого столетия [1] и существенно развита в работах Новожилова и его учеников [2] на основе метода низкоэнергетической бозонизации, успешно опробованного [3] в квантовой хромодинамике.

*Научно-исследовательский институт им. В. А. Фока, Санкт-Петербургский государственный университет, Санкт-Петербург, Россия. E-mail: andrian@heps.phys.spbu.ru; Vladimir.Andrianov@pobox.spbu.ru

${ }^{\dagger}$ University of Bologna, Bologna, Italy. E-mail: giacconi@bo.infn.it; soldati@bo.infn.it 
В настоящей работе исследуется возникновение индуцированной гравитации в $(4+1)$-мерном пространстве-времени и ее роль в образовании $(3+1)$-мерной Вселенной, локализованной на доме́нной стенке вследствие спонтанного нарушения трансляционной симметрии. Предположение о том, что наша Вселенная может существовать на доме́нной стенке (так называемой “бране”) в многомерном пространствевремени, вызвало недавно большой интерес [4], [5] благодаря тому, что эта идея открывает новые подходы к решению таких проблем, как происхождение массы и иерархия масштабов в теории элементарных частиц. Различные сценарии доме́нных стенок (бран) и их приложения можно найти в многочисленных обзорах [6]-[8].

Доме́нная стенка (брана) сама по себе часто рассматривается как элементарный геометрический объект в пространстве высших размерностей с нулевой протяженностью в дополнительных измерениях, ограничивающий суперструну и имеющий свою собственную динамику (см., например, работы [7] и ссылки в них).

С другой стороны, доме́нные стенки могут возникать как классические решения в эффективной теории поля в результате нарушения некоторой дискретной симметрии и, далее, трансляционной инвариантности. В частности, возникновение доме́нной стенки (или толстой браны) и захват ею легких частиц могут быть обусловлены [9]-[11] специальными фоновыми вакуумными полями в пространстве высших размерностей.

В связи с этим представляют интерес механизмы того, как такие фоновые поля могут образовываться и индуцировать спонтанное нарушение трансляционной симметрии. В частности, заслуживает внимания возможность образования доме́нной стенки вследствие самодействия некоторых частиц в пространстве высших измерений [12]. В этой работе мы продолжаем исследование некомпактной $(4+1)$-мерной фермионной модели с локальным сильным четырехфермионным взаимодействием, дополненной индуцированым фоновым гравитационным полем [13]. По сравнению с работой [13] здесь мы рассматриваем более реалистическое четырехфермионное взаимодействие, в результате которого фермионы Стандартной модели приобретают раличные массы в соответствии с феноменологией.

Оба типа взаимодействия участвуют в нарушении дискретной и трансляционной симметрий за счет образования вакуумного состояния в форме доме́нной стенки [14]. В результате топологического механизма захвата легкие массивные дираковские частицы остаются в обычном $(3+1)$-пространстве-времени. Тот же механизм приводит к локализованным модам для составных скалярных полей, дополняя фермионную материю на бране скалярной. Поскольку нарушение трансляционной симметрии происходит спонтанно, то в силу теоремы Голдстоуна некоторые скалярные поля оказываются безмассовыми (так называемые "браноны" [15]).

В разделе 2 формулируется пятимерная модель для фермионов с самодействием в каналах со спином 0 и 2. Чтобы локализовать дираковские фермионы и снабдить их массой, оказывается вполне достаточным четырехфермионное самодействие в скалярном канале. Оно содержит две размерные константы взаимодействия, выраженные в единицах масштаба $\Lambda$, который играет роль параметра "обрезания", ограничивающего энергию-импульс для виртуальных фермионов. Взаимодействие 
в канале со спином 2 (гравитация в пятимерном пространстве-времени) вводится нелинейно [13], обеспечивая ковариантность модели с помощью диффеоморфизмов пространства-времени, и дополняется затравочной космологической константой так, чтобы сбалансировать образование физической космологической константы на бране. Что касается гравитации, то она полностью порождается пятимерным веществом и, тем самым, является индуцированной гравитацией в смысле ЗельдовичаНовожилова-Сахарова.

В этом же разделе с помощью скалярной бозонизации четырехфермионного взаимодействия получено низкоэнергетическое эффективное действие для составных скалярных и гравитационных полей в приближении среднего поля (или большого числа $N$ фермионных степеней свободы в Стандартной модели). Часть скалярного четырехфермионного взаимодействия, ответственная за локализацию на бране, вводится универсально для всех фермионов, поскольку конфигурация браны является геометрическим объектом, не зависящим от флейворов. Другая же часть содержит константы четырехфермионного взаимодействия, различные для разных фермионов, чтобы после локализации наделить фермионы разными массами. Эффективное действие, возникающее из однопетлевых фермионных поправок, включает в себя эффективный потенциал с радиационными вкладами от высокоэнергетических виртуальных фермионов, который затем служит для описания низкоэнергетического явления спонтанного нарушения симметрии. Оно также содержит кинетические члены для скалярных и гравитационных полей, наделяя их структурой составных полей. При вычислении эффективного действия был использован метод конечномодовой регуляризации [16] с разделением фермионных полей на высоко- и низкоэнергетические компоненты в евклидовом пространстве-времени с инвариантным обрезанием.

В разделе 3 на основе анализа низкоэнергетического эффективного действия исследованы классические вакуумные конфигурации для гравитационных и скалярных полей. Наше исследование ограничивается классом конформно-подобных метрик (так называемых “линейчатых геометрий”) с гиперплоскостями пространства Минковского в каждой точке вдоль пятимерной координаты.

Найдено общее решение для уравнений Эйнштейна и нелинейных уравнений Клейна-Фока-Гордона в приближении слабой гравитации, т.е. в предположении относительно малой пятимерной гравитационной константы. Это приближение обосновано в разделе 4 при помощи нормировки на ньютоновскую гравитационную константу на доме́нной стенке. Оказывается, что в главном порядке по гравитационной константе уравнения движения для скалярных полей не чувствительны к гравитации, а их решения совпадают со случаем, исследованным в работе [12] для плоского пятимерного пространства-времени. В этом смысле в обсуждаемой модели брана и "свернутая" геометрия порождаются главным образом веществом, а не самодействием гравитации.

Пространственно-неоднородное решение уравнений Клейна-Фока-Гордона содержит масштаб $M$ нарушения трансляционной симметрии, обратная величина которого характеризует толщину браны и степень локализации легких частиц. Предпо- 
лагается, что он меньше, чем масштаб композитности $\Lambda$, что позволяет исследовать образование браны и явление локализации в области низких энергий. В свою очередь, уравнения типа Эйнштейна могут быть преобразованы так, что одно из них содержит лишь конформный метрический фактор пятимерного пространства антиде Ситтера $\left(A d S_{5}\right)$, в то время как другое уравнение представляет собой интеграл движения с космологической константой в качестве константы интегрирования.

В разделе 4 исследуются спектральные свойства легких частиц, локализованных на бране, и приводится структура низкоэнергетического лагранжиана. В главном порядке по пятимерной гравитационной константе вычислен спектр масс для легких частиц и их констант взаимодействий. Оказывается, что константы связи легких частиц параметризуются отношением $M / \Lambda$ (отношением масштабов нарушения симметрии и композитности) и тем самым при большой разнице этих масштабов скалярные поля отщепляются от других полей материи.

В разделе 5 проведена оценка различных масштабов и констант взаимодействий, введенных в работе, и найдены соотношения между пятимерной массой Планка, масштабами нарушения симметрий и кривизной пространства анти-де Ситтера $(A d S)$. Основой для этого служат значение ньютоновской константы и ограничения на отклонение от закона Ньютона на основе современных экспериментальных данных [17]. В сценарии индуцированной гравитации масштаб композитности оказывается значительно больше, чем масштаб нарушения трансляционной симметрии, и сравним с масштабом великого обьединения $10^{15}$ ГэВ. Поэтому, если эффективное гравитационное действие при сверхнизких энергиях преимущественно индуцируется фермионами, то скалярное взаимодействие сильно подавлено, и флуктуации браны (браноны) являются хорошими “стерильными” кандидатами для объяснения темной материи [15] (или темной энергии, если они безмассовые). Вычислена индуцированная космологическая константа на бране, которая точно обращается в нуль, обеспечивая самосогласованность с выбранным классом метрик с плоским пространством Минковского на бране.

В разделе 6 обсуждаются характерные черты индуцированной гравитации в пятимерном пространстве и ее следствия для параметров Вселенной на бране.

\section{2. МОДЕЛЬ ФЕРМИОНОВ В ПЯТИМЕРНОМ ПРОСТРАНСТВЕ-ВРЕМЕНИ С САМОДЕЙСТВИЕМ}

Напомним феноменологию доме́нной стенки [6], [12] и введем необходимые для этого обозначения. Начнем с модели одного четырехкомпонентного фермионного биспинорного поля $\psi(X)$, определенного в пятимерном плоском пространстве-времени Минковского и взаимодействующего со скалярным полем $\Phi(X)$.

Пятимерные координаты определяются следующим образом:

$$
X^{A}=\left(x^{\mu}, z\right), \quad x^{\mu}=\left(x^{0}, x^{1}, x^{2}, x^{3}\right), \quad\left(\eta^{A A}\right)=(+,-,-,-,-),
$$

где подпространство координат $x^{\mu}$ естественным образом соответствует четырехмерному пространству Минковского. Размер дополнительного пятого измерения предполагается бесконечным (или достаточно большим). Фермионная волновая функция 
описывается уравнением Дирака:

$$
\left[i \gamma^{A} \partial_{A}-\Phi(X)\right] \psi(X)=0, \quad \gamma^{A}=\left(\gamma^{\mu}, i \gamma_{5}\right), \quad\left\{\gamma^{A}, \gamma^{B}\right\}=2 \eta^{A B},
$$

где $\gamma^{\mu}$ образуют стандартный набор четырехмерных матриц Дирака в киральном (или вейлевском) представлении с $\gamma_{5} \equiv i \gamma^{0} \gamma^{1} \gamma^{2} \gamma^{3}$.

Захват легких фермионов на четырехмерной гиперплоскости, т.е. на доме́нной стенке, локализованной в пятом измерении, при $z=z_{0}$ может произойти вследствие определенной топологической конфигурации вакуумного ожидания для скалярного поля, например

$$
\langle\Phi(X)\rangle_{0}=\varphi(z)=M \operatorname{th}(M z),
$$

благодаря появлению нулевых мод с определенной киральностью в спектре четырехмерного оператора Дирака [4], [6]. Чтобы построить легкие дираковские фермионы, необходимо иметь две разные киральности для одной и той же конфигурации скалярного (фонового) поля. Тогда минимальный набор фермионов в пятимерном пространстве-времени должен включать в себя [10], [12] два фермиона $\psi_{1}(X), \psi_{2}(X)$, а чтобы образовать левые и правые части четырехмерных дираковских биспиноров из нулевых мод, эти фермионы должны взаимодействовать со скалярным полем $\Phi(X)$ с противоположными зарядами:

$$
\left[i \not \partial-\tau_{3} \Phi(X)\right] \Psi(X)=0, \quad \not \partial \equiv \widehat{\gamma}^{A} \partial_{A}, \quad \Psi(X)=\left(\begin{array}{c}
\psi_{1}(X) \\
\psi_{2}(X)
\end{array}\right),
$$

где $\widehat{\gamma}^{A} \equiv \gamma^{A} \otimes \mathbf{1}_{2}$ - дираковские матрицы, а $\tau_{a} \equiv \mathbf{1}_{4} \otimes \sigma_{a}, \quad a=1,2,3$, являются обобщениями матриц Паули для биспинорных компонент поля $\psi_{i}(X)$.

В дополнение к скалярному полю, обеспечивающему локализацию, требуется еще одно скалярное поле, чтобы снабдить массами фермионы на доме́нной стенке. Его взаимодействие с фермионами должно смешивать левые и правые киральности, поскольку массовый член нарушает киральную инвариантность. Таким образом, мы вводим два типа четырехфермионного самодействия, которые порождают два составных скалярных поля с подходящими константами связи для фермионов. Одно из них отвечает за локализацию на доме́нной стенке и тем самым взаимодействие его с фермионами предполагается универсальным, не зависящим от флейворов. Другое же участвует в формировании фермионных масс, и поэтому константы Юкавы его взаимодействия с различными фермионами должны быть разными. Аналогично фермионам в вакууме доме́нной стенки эти два скалярных поля приобретают легкие локализованные состояния. Динамический механизм захвата частиц на доме́нной стенке оказывается довольно предсказательным. В частности, в работе [12] были получены оценки для масс и констант распадов фермионов и бозонов. В данной работе выбран более реалистический сценарий с различными константами Юкавы для разных фермионов, и поэтому оценки для спектральных параметров частиц количественно иные, отличные от полученных в работе [12].

На основе предыдущего обсуждения определим фермионную модель в пяти измерениях, которая обеспечивает механизм нарушения трансляционной симметрии, 
приводящий к образованию доме́нной стенки и локализации легких массивных частиц. Будучи неперенормируемой, она может рассматриваться как эффективная - результат редукции более фундаментальной теории от планковских масштабов до масштаба композитности $\Lambda$. Модель определяется плотностью классического лагранжиана

$$
\begin{array}{r}
\mathcal{L}^{(5)}\left(\bar{\Psi}_{j}, \Psi_{j}\right)=\sum_{j=1}^{N_{f}} \bar{\Psi}_{j} i \not \partial \Psi_{j}+\frac{g_{1}}{4 N \Lambda^{3}}\left(\sum_{j=1}^{N_{f}} \bar{\Psi}_{j} \tau_{3} \Psi_{j}\right)^{2}+ \\
+\frac{1}{4 N \Lambda^{3}} \sum_{j, k=1}^{N_{f}} G_{2, j k l m} \bar{\Psi}_{j} \tau_{1} \Psi_{k} \bar{\Psi}_{l} \tau_{1} \Psi_{m}
\end{array}
$$

где $\Psi_{j}(X)$ - восьмикомпонентное пятимерное фермионное поле (см. уравнение $(2)$ ) с флейвором $j$, которое также может быть “цветным" мультиплетом с $N_{\text {c }}$ степеней свободы. Заметим, что введенное полное число $N$ цветных и флейворных степеней свободы массивных фермионов в Стандартной модели порядка двадцати, если все они порождаются соответствующими пятимерными фермионами. Будем считать нейтрино массивными дираковскими частицами; тогда $N=24$.

Ультрафиолетовый масштаб обрезания $\Lambda$ ограничивает импульсы фермионов, поскольку четырехфермионное взаимодействие предполагается эффективным, и соответственно константы эффективного взаимодействия $g_{1}$ и $G_{2, j k l m}$ безразмерны. Выберем структуру матрицы констант взаимодействия $G_{2, j k l m}$ в минимальной форме, которая достаточна для динамического образования масс фермионов, $G_{2, j k l m}=$ $g_{2, j} g_{2, l} \delta_{j k} \delta_{l m}$, и пренебрежем переходами с изменением флейворов. Тогда плотность лагранжиана (3) может быть бозонизирована при помощи пары внешних скалярных полей $\Phi(X)$ и $H(X)$,

$$
\mathcal{L}^{(5)}\left(\bar{\Psi}_{j}, \Psi_{j}, \Phi, H\right)=\sum_{j=1}^{N_{f}} \bar{\Psi}_{j}\left(i \not \partial-\tau_{3} \Phi-\tau_{1} \bar{g}_{j} H\right) \Psi_{j}-\frac{N \Lambda^{3}}{g_{1}} \Phi^{2}-\frac{N \Lambda^{3}}{g_{2}} H^{2} .
$$

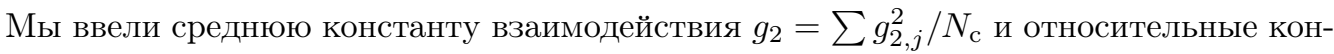
станты $\bar{g}_{j}=g_{2, j} / \sqrt{g_{2}}$. Сумма в определении константы $g_{2}$ распространяется как на флейворные, так и на цветные степени свободы. Эти константы в конечном итоге параметризуют массы фермионов в Стандартной модели. Поскольку константа Юкавы для топ-кварка значительно превышает соответствующие константы для всех остальных фермионов, то в хорошем приближении $g_{2} \simeq g_{t}^{2}$, а относительную константу в топ-канале в этом же приближении можно считать $\bar{g}_{t} \simeq 1$. Именно это приближение мы и будем использовать в дальнейших расчетах.

Для достаточно больших значений констант связи эта система испытывает фазовый переход в состояние, в котором конденсация фермион-антифермионных пар приводит к спонтанному нарушению $\tau$-симметрии:

$$
\begin{array}{ll}
\Psi_{j} \longrightarrow \tau_{1} \Psi_{j} ; & \Phi \longrightarrow-\Phi ; \\
\Psi_{j} \longrightarrow \tau_{3} \Psi_{j} ; & H \longrightarrow-H .
\end{array}
$$


Для того чтобы исследовать это явление, необходимо вычислить эффективную плотность лагранжиана, в которой учитываются степени свободы, существенные в инфракрасной области. Для этого перейдем в пространство Евклида, где определено инвариантное обрезание по импульсу. Для низкоэнергетических компонент будем считать, что импульсы $|p|<\Lambda_{0}$ малы по сравнению с обрезанием $\Lambda_{0} \ll \Lambda$. Разделим импульсное пространство фермионных полей на высокоэнергетическую $\Psi_{j}^{(\mathrm{h})}(p) \equiv \Psi_{j}(p) \vartheta\left(|p|-\Lambda_{0}\right) \vartheta(\Lambda-|p|)$ и низкоэнергетическую $\Psi_{j}^{(1)}(p) \equiv \Psi_{j}(p) \vartheta\left(\Lambda_{0}-|p|\right)$ части и проинтегрируем по высокоэнергетической части спектра фермионов $(\vartheta(t)$ - функция Хевисайда). Подобное разделение спектра фермионов должно быть выполнено ковариантно, т.е. для полного нормального оператора Дирака в евклидовом пространстве:

$$
\not D_{j} \equiv i\left(\not \partial+\tau_{3} \Phi+\tau_{1} \bar{g}_{j} H\right) .
$$

Поскольку мы исследуем инфракрасное нарушение $\tau$-симметрии, то можно пренебречь высокоэнергетическими компонентами внешних бозонных полей, что эквивалентно приближению среднего поля или большого числа $N$. В результате плотность лагранжиана, содержащая низкоэнергетические фермионные и бозонные поля, будет состоять из суммы классической плотности лагранжиана (4) и индуцированной однопетлевой части, возникающей после функционального интегрирования высокоэнергетических компонент фермионных полей, а именно

$$
\mathcal{L}_{\text {low }}^{(5)}\left(\bar{\Psi}_{j}^{(1)}, \Psi_{j}^{(1)}, \Phi, H\right)=\sum_{j=1}^{N_{f}} \bar{\Psi}_{j}^{(1)} \not D_{j} \Psi_{j}^{(1)}+\frac{N \Lambda^{3}}{g_{1}} \Phi^{2}+\frac{N \Lambda^{3}}{g_{2}} H^{2}+\Delta \mathcal{L}^{(5)}(\Phi, H) .
$$

Однопетлевой вклад от высокоэнергетических фермионов оказывается равным

$$
\Delta \mathcal{L}^{(5)}(\Phi, H)=-\frac{1}{2} \operatorname{tr}\left\langle X\left|\vartheta\left(\Lambda^{2}-\not D_{j}^{\dagger} \not D_{j}\right) \ln \frac{\not D_{j}^{\dagger} \not D_{j}}{\Lambda^{2}}-\vartheta\left(\Lambda_{0}^{2}-\not D_{j}^{\dagger} \not D_{j}\right) \ln \frac{\not D_{j}^{\dagger} \not D_{j}}{\Lambda_{0}^{2}}\right| X\right\rangle .
$$

В этой формуле операция следа осуществляется по спинорным и внутренним степеням свободы. В выражении (6) мы ввели обрезания, которые инвариантно выделяют ранее упомянутую высокоэнергетическую область [16]. Для $n=5$ главный вклад в плотность лагранжиана [12] приведен ниже.

В настоящей работе мы исследуем динамический механизм самодействия фермионов, при котором гравитационное действие Гильберта-Эйнштейна полностью индуцируется высокоэнергетическим спинорным полем. Наш основной интерес сосредоточен на образовании “толстой" браны, которая в пределе нулевой толщины воспроизводит плоское $(3+1)$-мерное пространство Минковского. В направлении пятого измерения, ортогонального к пространству Минковского, гравитация оказывается нетривиальной, если вакуумные конфигурации для составных скалярных полей нарушают трансляционную инвариантность.

Введем гравитацию на пятимерном римановом многообразии $\mathcal{M}_{5}$ при помощи метрического поля $g_{A B}(X)$. В классическом действии $S$ инвариантная мера в интеграле по многообразию нормирована на определитель этой метрики $\mathbf{g} \equiv \operatorname{det}\left(g_{A B}\right)$,

$$
S\left(\Phi, H, \bar{\Psi}_{j}^{(1)}, \Psi_{j}^{(1)}, \mathbf{g}\right)=\int_{\mathcal{M}_{5}} d^{5} X \sqrt{\mathbf{g}}\left[\mathcal{L}_{\text {fermion }}^{(5)}+\mathcal{L}_{\text {boson }}^{(5)}\right]
$$


а фермионная (спинорная) и бозонная (скалярная и гравитационная) части плотности лагранжиана определены ниже.

В уравнении (7) плотность лагранжиана, билинейная по фермионным полям и инвариантная относительно диффеоморфизмов, может быть определена в терминах полей пентад $e_{A}^{i}(X)$, которые локально связывают криволинейное многообразие $\mathcal{M}_{5}$ и плоское пространство с евклидовой сигнатурой. Латинские индексы, нумерующие базис упомянутого плоского пространства, принимают значения от единицы до пяти так, что алгебра обобщенных матриц Дирака имеет вид

$$
\left\{\widehat{\gamma}_{j}, \widehat{\gamma}_{k}\right\}=2 \delta_{j k}\left(\mathbf{1}_{4} \otimes \mathbf{1}_{2}\right)
$$

В этом случае инвариантная плотность лагранжиана для спинорных полей может быть определена как

$$
\begin{aligned}
\mathcal{L}_{\text {fermion }}^{(5)}\left(\bar{\Psi}_{j}^{(1)}, \Psi_{j}^{(1)}, \Phi, H, \mathbf{g}\right) & =i \sum_{j=1}^{N_{f}} \bar{\Psi}_{j}^{(1)}\left[\widehat{\gamma}_{k} e_{k}^{A}\left(\partial_{A}+\omega_{A}\right)+\tau_{3} \Phi+\tau_{1} \bar{g}_{j} H\right] \Psi_{j}^{(\mathrm{l})}= \\
& =i \sum_{j=1}^{N_{f}} \bar{\Psi}_{j}^{(\mathrm{l})}\left(\not \nabla+\tau_{3} \Phi+\tau_{1} \bar{g}_{j} H\right) \Psi_{j}^{(\mathrm{l})} \equiv \sum_{j=1}^{N_{f}} \bar{\Psi}_{j}^{(1)} \mathbf{D}_{j} \Psi_{j}^{(\mathrm{l})},
\end{aligned}
$$

а спин-связность $\omega_{A}$ выражается в терминах пентад и аффинной связности $\Gamma_{A B}^{C}$ :

$$
\omega_{A} \equiv \frac{1}{8}\left[\widehat{\gamma}_{i}, \widehat{\gamma}_{j}\right] \Gamma_{A}^{i j}, \quad \Gamma_{A}^{i j}=e^{B j} \Gamma_{A B}^{C} e_{C}^{i}-e^{B j} \partial_{A} e_{B}^{i}
$$

Как и ранее в выражении (5), фермионное самодействие порождает вершины типа Юкавы, которые линейны по внешним скалярным полям $\Phi$ и $H$. Напомним, что фаза с нарушением симметрии возникает, когда классическое скалярное взаимодействие компенсирует большие вклады $\sim \Lambda^{3}$ в низкоэнергетическом эффективном действии (6), индуцированном высокоэнергетическими фермионами. Введение гравитации приводит к большому вкладу $\sim \Lambda^{5}$ в однопетлевое действие, который должен быть компенсирован так, чтобы гравитационная динамика была не слишком подавлена большой космологической константой.

Для компенсации больших однопетлевых вкладов, которые индуцированы фермионным веществом, необходимо добавить классическую бозонную плотность лагранжиана с большими (затравочными) коэффициентами подходящей величины и знака:

$$
\mathcal{L}_{\text {boson }}^{(5)}(\Phi, H, \mathbf{g})=N \Lambda^{3}\left(\frac{\Phi^{2}}{g_{1}}+\frac{H^{2}}{g_{2}}\right)+\Lambda^{3} \lambda_{0} .
$$

В этом уравнении величина $\lambda_{0} \sim \Lambda^{2}$ является затравочной космологической константой пятимерной Вселенной. В нашем подходе гравитационная плотность лагранжиана Гильберта-Эйнштейна полностью индуцируется фермионным веществом и не появляется в затравочном действии. Тем самым на масштабах композитности гравитация оказывается очень сильной и, строго говоря, ее описание требует последовательного квантования. Однако в низкоэнергетической области гравитационная 
плотность лагранжиана приобретает большой коэффициент как результат вакуумных вкладов высокоэнергетических фермионов, и, следовательно, пятимерные гравитационные поля становятся очень слабыми. Для этого сценария далее мы оценим соответствующие масштабы пятимерной физики, обеспечивающей обычную ньютоновскую гравитацию в нашей Вселенной, расположенной на бране.

Вычислим низкоэнергетическое эффективное действие в искривленном пятимерном пространстве в присутствии гравитации. Для этой цели определим эллиптический оператор второго порядка, имеющий тот же спектр, что и оператор Дирака в формуле (9). Для его построения используем свойство сопряжения $\boldsymbol{D}_{j}^{\dagger}=\tau_{2} \boldsymbol{D}_{j} \tau_{2}$, которое связывает спектры ковариантного оператора Дирака и его эрмитово сопряженного. Благодаря этому ковариантный оператор Дирака является нормальным оператором, что позволяет получить вещественное эффективное действие и определить обрезание спектра при помощи положительного оператора [18]:

$$
\begin{aligned}
\not_{j}^{\dagger} \not_{j} & =(i \not \nabla)^{2}+\Phi^{2}(X)+\bar{g}_{j}^{2} H^{2}(X)-\tau_{3} \not \partial \Phi(X)-\tau_{1} \bar{g}_{j} \not \partial H(X)= \\
& =-g^{A B}(X) D_{A} D_{B}+\mathcal{M}_{j}^{2}(X) \equiv-D^{2}+\mathcal{M}_{j}^{2}(X) .
\end{aligned}
$$

Полная ковариантная производная от $f$ определяется как $D_{A} f^{C}=\left(\partial_{A}+\omega_{A}\right) f^{C}+$ $\Gamma_{A B}^{C} f^{B}$, а оператор $\mathcal{M}_{j}^{2}(X)$ является матричным мультипликативным оператором,

$$
\mathcal{M}_{j}^{2}(X)=\frac{R}{4}+\Phi^{2}(X)+\bar{g}_{j}^{2} H^{2}(X)-\tau_{3} \not \partial \Phi(X)-\tau_{1} \bar{g}_{j} \not \partial H(X) .
$$

При вычислении эффективной лагранжевой плотности, которая индуцирована высокоэнергетическими фермионами, вклады дает только масштабная аномалия $\Delta \mathcal{L}^{(5)}$, а именно та ее часть, которая зависит от степеней масштаба обрезания:

$$
\Delta \mathcal{L}^{(5)}(\Phi, H, \mathbf{g})=\int_{\Lambda_{0}}^{\Lambda} \frac{d Q}{Q} \operatorname{tr}\left\langle X\left|\vartheta\left(Q^{2}-\boldsymbol{D}_{j}^{\dagger} \boldsymbol{D}_{j}\right)\right| X\right\rangle .
$$

Мы предполагаем, что скалярные поля медленно меняются и тем самым несут импульс много меньший, чем масштаб низких энергий $\Lambda_{0}$. Поэтому диагональный матричный элемент в правой части уравнения (14) может быть вычислен при помощи разложения по производным [16]. В градиентном разложении [19] нулевой и первый порядки коэффициентов Сили-Джилки $a_{0}, a_{2}$ содержат соответственно инвариантную меру $\sqrt{\mathbf{g}}$ и скалярную кривизну $R[20]$. Принимая во внимание, что $\Lambda_{0} \ll \Lambda$, можно пренебречь зависимостью от $\Lambda_{0}$ и с точностью до полной пятимерной производной получить следующее выражение:

$$
\begin{gathered}
\Delta \mathcal{L}^{(5)}(\Phi, H, \mathbf{g}) \stackrel{\Lambda \rightarrow \infty}{\sim} \frac{N \Lambda^{5}}{75 \pi^{3}}-\frac{\Lambda^{3}}{9 \pi^{3}}\left\{N\left(\Phi^{2}+\frac{R}{12}\right)+N_{\mathrm{c}} H^{2}\right\}+\Lambda a_{4}, \\
a_{4}=\frac{1}{4 \pi^{3}}\left\{N \partial_{A} \Phi \partial^{A} \Phi+N_{\mathrm{c}} \partial_{A} H \partial^{A} H+N \Phi^{4}+2 N_{\mathrm{c}} \Phi^{2} H^{2}+N_{\mathrm{c}} H^{4}+\right. \\
\left.+\frac{R}{6}\left(N \Phi^{2}+N_{\mathrm{c}} H^{2}\right)+N\left(\frac{R^{2}}{144}-\frac{1}{90} R_{A B} R^{A B}-\frac{7}{720} R_{A B C D} R^{A B C D}\right)\right\} .
\end{gathered}
$$


В этом уравнении $a_{4}$ - четвертый коэффициент Сили-Джилки данного градиентного разложения, $R_{A B C D}$ является тензором римановой кривизны, а $R_{A B}$ - тензором Риччи [20]. Хотя некоторые значения коэффициентов могут быть регуляризационно зависимы, относительные коэффициенты для кинетических членов и членов взаимодействия четвертой степени в эффективной низкоэнергетической плотности лагранжиана оказываются универсальными по отношению к регуляризациям. При вычислении следов были использованы следующие оценки сумм: $\sum \bar{g}_{j}^{2} \simeq \sum \bar{g}_{j}^{4} \simeq N_{\mathrm{c}}$.

\section{3. КЛАССИЧЕСКИЕ ВАКУУМНЫЕ КОНФИГУРАЦИИ ПОЛЕЙ}

В этой модели возникают различные динамические режимы с нарушением $\tau$ симметрии и трансляционной инвариантности в зависимости от соотношения размерных констант взаимодействия низкоэнергетической плотности лагранжиана (5). Полная низкоэнергетическая евклидова плотность лагранжиана может быть записана в виде

$$
\begin{aligned}
\mathcal{L}_{\text {low }}^{(5)} \equiv & \mathcal{L}_{\text {fermion }}^{(5)}\left(\bar{\Psi}^{(1)}, \Psi^{(1)}, \Phi, H, \mathbf{g}\right)+\mathcal{L}_{\text {boson }}^{(5)}(\Phi, H, \mathbf{g})+\Delta \mathcal{L}^{(5)}(\Phi, H, \mathbf{g})= \\
= & \left.i \sum_{j=1}^{N_{f}} \bar{\Psi}_{j}^{(1)}(X)[\not]+\tau_{3} \Phi(X)+\tau_{1} \bar{g}_{j} H(X)\right] \Psi_{j}^{(1)}(X)+ \\
& \quad+\frac{\Lambda}{4 \pi^{3}}\left\{-2 N \Delta_{1} \Phi^{2}(X)-2 N_{\mathrm{c}} \Delta_{2} H^{2}(X)\right\}-\frac{N \Lambda^{3}}{108 \pi^{3}}\{R(X)-2 \lambda\}+\Lambda a_{4},
\end{aligned}
$$

где два массовых параметра $\Delta_{i}$ характеризуют отклонение от критической точки $g_{j}^{\mathrm{cr}}$ :

$$
\begin{array}{rlrl}
\Delta_{1}\left(g_{1}\right) & =\frac{2 \Lambda^{2}}{9 g_{1}}\left(g_{1}-g_{1}^{\mathrm{cr}}\right), & & g_{1}^{\mathrm{cr}}=9 \pi^{3} ; \\
\Delta_{2}\left(g_{t}\right)=\frac{2 \Lambda^{2}}{9 g_{t}^{2}}\left(g_{t}^{2}-g_{2}^{\mathrm{cr}}\right), & g_{2}^{\mathrm{cr}}=\frac{9 N \pi^{3}}{N_{\mathrm{c}}} .
\end{array}
$$

Перенормированная космологическая константа

$$
\lambda=\lambda_{0}+\frac{18 \Lambda^{2}}{25}
$$

возникает в результате баланса между классическим и индуцированным вкладами, а гравитационный пятимерный масштаб Планка

$$
M_{*}^{3} \equiv \frac{N \Lambda^{3}}{54 \pi^{3}}
$$

полностью индуцируется высокоэнергетическими фермионами. Поскольку затравочное гравитационное действие отсутствует, то мы приходим к сценарию индуцированной гравитации. Мы ожидаем, что основные физические масштабы меньше, чем пятимерная масса Планка, иначе наша эффективная модель должна быть заменена на все еще не известную теорию квантовой гравитации.

Следуя этому, предположим, что эффективные константы связи вещества приводят к низкоэнергетическому масштабу $M \ll \Lambda$, который определяется величиной энергетической щели $\Delta_{1}=M^{2}$. Чтобы различные части уравнений движения 
были соизмеримы по величине, необходимо соответствующим образом подобрать константы связи в нашей модели. В частности, для обеспечения малости $\lambda \ll \Lambda^{2}$ затравочная космологическая константа связи должна быть отрицательной: $\lambda_{0}<0$.

Получим решения типа браны, которые описывают локализацию частиц в окрестности какой-либо точки по пятой координате $z$. В настоящей работе нас интересуют лишь статические эффекты удержания частиц на бране, а космологическую эволюцию мы не рассматриваем. Именно поэтому мы ожидаем, что локализованные частицы будут распространяться в плоском четырехмерном пространстве-времени (для упрощения расчетов - с евклидовой сигнатурой). Таким образом, ограничимся анализом квазиплоской римановой метрики, инвариантный линейный элемент которой выберем в виде

$$
d s^{2}=g_{A B}(X) d X^{A} d X^{B}=e^{-2 \rho(z)} d x_{\mu} d x_{\mu}+d z^{2} .
$$

Соответствующий инвариантный элемент объема $-\sqrt{\mathbf{g}}=e^{-4 \rho(z)}$, а скалярная и гравитационная части низкоэнергетической лагранжевой плотности (16) для этой метрики принимают следующую форму:

$$
\begin{aligned}
\mathcal{L}_{\text {boson }}^{(5)}(\Phi, H, \mathbf{g}) & +\Delta \mathcal{L}^{(5)}(\Phi, H, \mathbf{g})= \\
= & \frac{\Lambda}{4 \pi^{3}}\left\{e^{2 \rho(z)}\left[N \partial_{\mu} \Phi(X) \partial_{\mu} \Phi(X)+N_{\mathrm{c}} \partial_{\mu} H(X) \partial_{\mu} H(X)\right]+\right. \\
& +N\left[\partial_{z} \Phi(X)\right]^{2}+N_{\mathrm{c}}\left[\partial_{z} H(X)\right]^{2}-2 N \Delta_{1} \Phi^{2}(X)-2 N_{\mathrm{c}} \Delta_{2} H^{2}(X)+ \\
& \left.+N \Phi^{4}(X)+2 N_{\mathrm{c}} \Phi^{2}(X) H^{2}(X)+N_{\mathrm{c}} H^{4}(X)\right\}+ \\
& +\frac{\Lambda}{3 \pi^{3}}\left[N \Phi^{2}(X)+N_{\mathrm{c}} H^{2}(X)\right]\left\{\rho^{\prime \prime}(z)-\frac{5}{2}\left[\rho^{\prime}(z)\right]^{2}\right\}+ \\
& +\frac{N \Lambda^{3}}{54 \pi^{3}}\left\{-4 \rho^{\prime \prime}(z)+10\left[\rho^{\prime}(z)\right]^{2}+\lambda\right\}+ \\
& +\frac{N \Lambda}{120 \pi^{3}}\left\{2\left[\rho^{\prime \prime}(z)\right]^{2}-36 \rho^{\prime \prime}(z)\left[\rho^{\prime}(z)\right]^{2}+45\left[\rho^{\prime}(z)\right]^{4}\right\},
\end{aligned}
$$

где $\rho^{\prime}(z) \equiv d \rho / d z, \rho^{\prime \prime}(z) \equiv d^{2} \rho / d z^{2}$. Найдем классические решения для метрики (20) в режиме слабой связи с гравитационным полем. Для выбранной метрики последнее означает, что вдоль дополнительного измерения $\left|\rho^{\prime}(z)\right| / M=o(1),\left|\rho^{\prime \prime}(z)\right| / M^{2}=o(1)$. Тем самым конформная функция $\rho(z)$ медленно меняется на интервале порядка типичной комптоновской длины волны для низкоэнергетических частиц. Это условие совместно с $M \ll \Lambda$ приводит к тому, что члены, квадратичные по скалярной кривизне и тензору кривизны в плотности лагранжиана (16), в последнем слагаемом уравнения (21) оказываются несущественными.

В рамках приближения слабого гравитационного взаимодействия основная динамика определяется действием Эйнштейна-Гильберта на пятимерном римановом многообразии $\mathcal{M}_{5}$ и его связью со скалярными полями материи. Таким образом, уравнения поля могут быть записаны как

$$
R_{A B}-\frac{1}{2} g_{A B}(R-2 \lambda) \equiv G_{A B}+\lambda g_{A B}=\frac{27}{\Lambda^{2}} t_{A B}
$$


где нормированный тензор энергии-импульса для скалярных полей материи имеет вид

$$
\begin{aligned}
t_{A B} \equiv \frac{4 \pi^{3}}{N \Lambda} T_{A B} \equiv \partial_{A} \Phi \partial_{B} \Phi+\frac{N_{\mathrm{c}}}{N} \partial_{A} H \partial_{B} H-\frac{1}{2} g_{A B}\left(\partial_{C} \Phi \partial^{C} \Phi+\frac{N_{\mathrm{c}}}{N} \partial_{C} H \partial^{C} H-\right. \\
\left.\quad-2 \Delta_{1} \Phi^{2}-\frac{2 N_{\mathrm{c}}}{N} \Delta_{2} H^{2}+\Phi^{4}+\frac{2 N_{\mathrm{c}}}{N} \Phi^{2} H^{2}+\frac{N_{\mathrm{c}}}{N} H^{4}\right)+ \\
+\frac{1}{6}\left(R_{A B}-\frac{1}{2} g_{A B} R+g_{A B} D^{C} \partial_{C}-D_{B} \partial_{A}\right)\left(\Phi^{2}+\frac{N_{\mathrm{c}}}{N} H^{2}\right) .
\end{aligned}
$$

В свою очередь, уравнения движения для скалярных полей выглядят следующим образом:

$$
\begin{aligned}
2\left(\Delta_{1}-\Phi^{2}-\frac{N_{\mathrm{c}}}{N} H^{2}\right) \Phi & =\left(\frac{R}{6}-\frac{1}{\sqrt{g}} \partial_{C} \sqrt{g} g^{C D} \partial_{D}\right) \Phi \\
2\left(\Delta_{2}-H^{2}-\Phi^{2}\right) H & =\left(\frac{R}{6}-\frac{1}{\sqrt{g}} \partial_{C} \sqrt{g} g^{C D} \partial_{D}\right) H
\end{aligned}
$$

В случае квазиплоской метрики (20) и для классических конфигураций скалярных полей типа кинка $\langle\Phi(X)\rangle_{0}=\Phi(z),\langle H(X)\rangle_{0}=H(z)$ уравнения для скалярных полей материи приобретают вид

$$
\begin{aligned}
& \Phi^{\prime \prime}=2 \Phi\left(\Phi^{2}+\frac{N_{\mathrm{c}}}{N} H^{2}\right)-2 \Delta_{1} \Phi+4 \rho^{\prime} \Phi^{\prime}+\frac{2}{3} \Phi\left(2 \rho^{\prime \prime}-5 \rho^{2}\right), \\
& H^{\prime \prime}=2 H\left(\Phi^{2}+H^{2}\right)-2 \Delta_{2} H+4 \rho^{\prime} H^{\prime}+\frac{2}{3} H\left(2 \rho^{\prime \prime}-5 \rho^{2}\right) .
\end{aligned}
$$

Для дальнейшего удобно нормировать все размерные константы на низкоэнергетический масштаб масс $\Delta_{1}=M^{2}$. Это достигается введением безразмерного низкоэнергетического гравитационного параметра

$$
\kappa \equiv \frac{9 M^{2}}{\Lambda^{2}} \ll 1 .
$$

Из уравнений (22) можно получить уравнения для суммы диагональных компонент " $\mu \mu "+" 55 "$ "

$$
\rho^{\prime \prime}=\frac{\kappa}{M^{2}}\left\{\Phi^{\prime 2}+\frac{N_{\mathrm{c}}}{N} H^{\prime 2}+\frac{1}{2}\left(\rho^{\prime \prime}-\frac{1}{3} \frac{d^{2}}{d z^{2}}-\frac{1}{3} \rho^{\prime} \frac{d}{d z}\right)\left(\Phi^{2}+\frac{N_{\mathrm{c}}}{N} H^{2}\right)\right\},
$$

и для компоненты " 55 ":

$$
\begin{aligned}
\frac{2 M^{2}}{3 \kappa} \lambda=\Phi^{\prime 2} & +\frac{N_{\mathrm{c}}}{N} H^{\prime 2}+2 \Delta_{1} \Phi^{2}+\frac{2 N_{\mathrm{c}}}{N} \Delta_{2} H^{2}- \\
& -\Phi^{4}-\frac{2 N_{\mathrm{c}}}{N} \Phi^{2} H^{2}-\frac{N_{\mathrm{c}}}{N} H^{4}+ \\
& +\left(2 \rho^{\prime 2}-\frac{4}{3} \rho^{\prime} \frac{d}{d z}\right)\left(\Phi^{2}+\frac{N_{\mathrm{c}}}{N} H^{2}\right)-\frac{4 M^{2}}{\kappa} \rho^{\prime 2}
\end{aligned}
$$

Последнее уравнение в действительности представляет собой интеграл движения с пятимерной космологической константой в качестве константы интегрирования. 
Это можно проверить дифференцированием вышеупомянутого уравнения с учетом (25), (26) и (28). В результате мы имеем четыре уравнения для трех функций $\rho(z), \Phi(z), H(z)$ и одну константу интегрирования $\lambda_{\text {eff. }}$ Эти уравнения можно решить приближенно, разлагая по параметру $\kappa \ll 1$ и предполагая, что $\left|\rho^{\prime}(z)\right| / M=$ $\left|\rho^{\prime \prime}(z)\right| / M^{2}=O(\kappa)$. Разложение решений уравнений $(25),(26),(28)$ и $(29)$ по степеням $\kappa$ хорошо определено на всей длине пятого измерения, $-\infty<z<\infty$. В нулевом порядке по $\kappa$ можно найти [12] два типа решения для уравнений (25), (26). Однако в отличие от результатов работы [12] в уравнении (25) появляется второй малый параметр $N_{\mathrm{c}} / N=1 / 8 \ll 1$ для Стандартной модели. В главном приближении по этому параметру решения оказываются равными

$$
\begin{aligned}
(J): & \Phi_{J} \equiv\langle\Phi(X)\rangle_{0}=M \operatorname{th}(M z), \quad H_{J} \equiv\langle H(X)\rangle_{0}=0 ; \\
(K): & \Phi_{K} \equiv\langle\Phi(X)\rangle_{0}=M\left[\operatorname{th}(\beta z)+O\left(\frac{N_{\mathrm{c}} \mu^{2}}{N M^{2}}\right)\right], \\
H_{K} & \equiv\langle H(X)\rangle_{0}=\mu\left[\operatorname{sech}(\beta z)+O\left(\frac{\mu^{2}}{M^{2}}\right)\right],
\end{aligned}
$$

где введены обозначения

$$
\beta^{2}=M^{2}-\tilde{\mu}^{2}, \quad \tilde{\mu}^{2}=\frac{N_{\mathrm{c}}}{N} \mu^{2}=2 \Delta_{2}-M^{2} .
$$

Решение $(K)$ приведено в формуле (31) приближенно в разложении по параметру $\mu / M$, который характеризует отношение масштабов масс частиц Стандартной модели (см. раздел 4) к масштабу нарушения трансляционной инвариантности. Чтобы толщина браны не влияла на кинематику локализованных частиц, необходимо, чтобы их комптоновские длины волн были значительно больше, чем толщина доме́нной стенки, т.е. $\mu \ll M$. Именно это приближение и используется в дальнейшем. Легко видеть, что решение $(K)$ существует только в интервале $\Delta_{2}<M^{2}<2 \Delta_{2}$ и совпадает с экстремумом решения $(J)$ в пределе $\Delta_{2} \rightarrow M^{2} / 2, \mu \rightarrow 0$. Оба решения дают согласованно

$$
\lambda=\frac{27 M^{4}}{2 \Lambda^{2}}=\frac{3}{2} \kappa M^{2} .
$$

Из уравнения (28) можно найти конформный фактор в следующей форме:

$$
\rho(z) \simeq \frac{2 \kappa}{3} \ln \operatorname{ch}(M z)+B z .
$$

Это решение нормировано так, чтобы для нулевой константы интегрирования $B \rightarrow 0$ функция $\rho(z)$ становилась четной и $\rho(0)=0$. Последнее свойство отвечает нормировке метрики в $(3+1)$-пространстве на бране в точке $z=0$. Очевидно, что, когда $B=0$, это решение воспроизводит симметричную метрику пространства $A d S_{5}$ при больших $z$, а именно

$$
\rho(z) \stackrel{|z| \rightarrow \infty}{\sim} k|z|, \quad k \approx \frac{2}{3} \kappa M
$$

$k$ характеризует кривизну пространства $A d S_{5}$, величина которой определяет отклонение от закона Ньютона на малых расстояниях [17]. 


\section{4. ФИЗИКА НА ДОМЕННОЙ СТЕНКЕ В СЕКТОРЕ ВЕЩЕСТВА}

В результате образования доме́нной стенки мы ожидаем, что локализованные фермионы Стандартной модели приобретут массы $m_{j}^{(f)}$ много меньшие, чем низкоэнергетический масштаб $M$, т.е. естественным образом возникнет физика частиц в $(3+1)$-мерном пространстве-времени. Таким образом, в нашем рассмотрении возникают три масштаба $\mu \ll M \ll \Lambda$, из которых наименьший $\mu$ представляет собой масштаб физики сверхнизких энергий.

Опишем структуру спектра и взаимодействия частиц на бране в отсутствие гравитации. В окрестности вакуумного состояния (30) или (31) и точки скейлинга $M^{2}=\Delta_{1}=2 \Delta_{2}$ или $\mu=0$ кинетические операторы (вторые вариации действия) для двух скаляров $\Phi(X)$ и $H(X)$ и спинорных полей $\Psi_{j}(X)$ имеют нулевые моды, нормируемые в пятом измерении [12].

Эти нулевые моды $\phi_{0}(z), h_{0}(z)$ и $\psi_{0, j}(z)$ локализованы около $z=0$, имея толщину $\sim 1 / M$, и при сверхнизких энергиях флуктуации полей материи могут быть разложены следующим образом:

$$
\begin{aligned}
\Phi(X) & \simeq\langle\Phi(X)\rangle_{0}+\phi(x) \phi_{0}(z), & \phi_{0}(z) & \simeq \operatorname{sech}^{2}(M z) \sqrt{\frac{3 M \pi^{3}}{2 \Lambda N}} \\
H(X) & \simeq\langle H(X)\rangle_{0}+h(x) h_{0}(z), & h_{0}(z) & \simeq(\operatorname{sech}(M z))^{1-2 \epsilon} \sqrt{\frac{M \pi^{3}}{\Lambda N_{\mathrm{c}}}}, \quad \epsilon \equiv \frac{\mu^{2}}{M^{2}} \\
\Psi_{j}(X) & \simeq \psi_{j}(x) \psi_{0, j}(z), & \psi_{0, j}(z) & \simeq \operatorname{sech}(M z) \sqrt{\frac{M}{2}}
\end{aligned}
$$

Для этих конфигураций эффективная плотность лагранжиана (в пространстве Минковского) оказывается равной

$$
\begin{aligned}
\left.\mathcal{L}^{(4)}\right|_{\mu=0}= & \sum_{j=1}^{N_{f}} \bar{\psi}_{j}(x)\left(i \not \partial-g_{j}^{(\mathrm{Y})} h(x)\right) \psi_{j}(x)+\frac{1}{2}\left(\partial_{\mu} \phi(x)\right)^{2}+\frac{1}{2}\left(\partial_{\mu} h(x)\right)^{2}- \\
& -\lambda_{1} \phi^{4}(x)-\lambda_{2} \phi^{2}(x) h^{2}(x)-\lambda_{3} h^{4}(x)
\end{aligned}
$$

с эффективными константами сверхнизких энергий: Юкавы $g_{j}^{(\mathrm{Y})}-$ для фермионов и самодействия $\lambda_{k}$ - для скалярных полей типа Хиггса $h(x)$ и бранонов $\phi(x)$ :

$$
g_{j}^{(\mathrm{Y})}=\frac{\pi}{4} \bar{g}_{j} \sqrt{\frac{N \zeta}{N_{\mathrm{c}}}}, \quad \lambda_{1}=\frac{18}{35} \zeta, \quad \lambda_{2}=\frac{4}{5} \zeta, \quad \lambda_{3}=\frac{N}{3 N_{\mathrm{c}}} \zeta, \quad \zeta \equiv \frac{M \pi^{3}}{\Lambda N}=\frac{\pi^{3}}{3 N} \sqrt{\kappa}
$$

В присутствии гравитации [13] нулевые моды остаются локализованными, и поэтому вакуумное решение, соответствующее в асимптотике геометрии $A d S_{5}$, не играет существенной роли в определении констант в выражении (37).

Для вакумной конфигурации $(K)$ отклонение от точки скейлинга при $\mu \ll M$ порождает массы для частиц Хиггса и фермионов. Более того, появляются трили- 
нейные скалярные вершины:

$$
\begin{gathered}
\Delta \mathcal{L}_{\mu}^{(4)}=-\frac{1}{2} m_{h}^{2} h^{2}(x)-\sum_{j=1}^{N_{f}} m_{j}^{(f)} \bar{\psi}_{j}(x) \psi_{j}(x)-\lambda_{4} h^{3}(x)-\lambda_{5} \phi^{2}(x) h(x), \\
m_{h}^{2}=\mu^{2}\left(4-\frac{2 N_{\mathrm{c}}}{N}\right), \quad m_{j}^{(f)}=\frac{\pi}{4} \bar{g}_{j} \mu, \quad \lambda_{4}=\frac{4}{3} \mu \sqrt{\zeta \frac{N}{N_{\mathrm{c}}}}, \quad \lambda_{5}=\frac{8}{5} \mu \sqrt{\zeta \frac{N_{\mathrm{c}}}{N}} .
\end{gathered}
$$

Мы видим, что все вершины взаимодействия контролируются параметром $\zeta \sim M / \Lambda$, и если $\zeta \ll 1$, то скалярная материя практически не взаимодействует с фермионами и сама с собой (мы не рассматриваем эффекты калибровочных полей). Однако значения параметра $\zeta$ трудно предсказать в отсутствие гравитации, его величина может быть ограничена сверху лишь экспериментальными данными. С другой стороны, массы скаляров типа Хиггса и фермионов контролируются ультранизким масштабом $\mu$ независимо от $\zeta$.

Если следовать Стандартной топ-модели [21], то можно считать, что самый тяжелый топ-кварк $\bar{g}_{t}=1$ и дает основной вклад в динамическое нарушение симметрии, тем самым масштаб $\mu \sim m_{\text {top }} \sim 200$ ГэВ имеет порядок масштаба нарушения электрослабых взаимодействий.

\section{5. ОЦЕНКИ ПАРАМЕТРОВ МОДЕЛИ ИЗ НЬЮТОНОВСКОЙ ГРАВИТАЦИОННОЙ КОНСТАНТЫ}

Найдем соотношение между пятимерной гравитацией в $A d S_{5}$-мире и гравитацией на бране, используя факторизованную риманову метрику

$$
d s^{2}=e^{-2 \rho(z)} g_{\mu \nu}(x) d x^{\mu} d x^{\nu}+d z^{2} .
$$

Для этой метрики в главном порядке по параметру $\kappa$ эффективное четырехмерное действие Эйнштейна-Гильберта имеет вид

$$
\begin{aligned}
S[g]= & -\frac{N \Lambda^{3}}{108 \pi^{3}} \int d^{5} X \sqrt{\mathbf{g}(X)}\{R(X)-2 \lambda\} \simeq \\
\simeq & -\frac{N \Lambda^{3}}{108 \pi^{3}} \int d^{4} x \sqrt{\mathbf{g}(x)} R(x) \int_{-\infty}^{+\infty} d z e^{-2 \rho(z)}- \\
& \quad-\frac{N \Lambda^{3}}{54 \pi^{3}} \int d^{4} x \sqrt{\mathbf{g}(x)} \int_{-\infty}^{+\infty} d z e^{-4 \rho(z)}\left\{6\left[\rho^{\prime}(z)\right]^{2}-\lambda\right\} \equiv \\
\equiv & -\frac{1}{16 \pi G_{N}} \int d^{4} x \sqrt{\mathbf{g}(x)}\left\{R(x)-2 \Lambda_{\text {grav }}\right\},
\end{aligned}
$$

откуда мы в итоге получаем массу Планка $M_{\mathrm{P}} \sim 1.22 \times 10^{19} \Gamma \ni \mathrm{B} / \mathrm{c}^{2}$, которая соответствует ньютоновской гравитационной константе

$$
M_{\mathrm{P}}^{2}=G_{N}^{-1} \equiv \frac{4 N \Lambda^{3}}{27 \pi^{2}} \int_{-\infty}^{+\infty} d z e^{-2 \rho(z)} .
$$


В свою очередь, гравитационная часть четырехмерной космологической константы оказывается равной

$$
\Lambda_{\text {grav }} \equiv \frac{4 N G_{N} \Lambda^{3}}{27 \pi^{2}} \int_{-\infty}^{+\infty} d z e^{-4 \rho(z)}\left\{\lambda-6\left[\rho^{\prime}(z)\right]^{2}\right\} .
$$

Используя в уравнении (41) приближенное решение (34) при $\kappa \ll 1$ и $|z| \rightarrow \infty$, можно получить

$$
G_{N} \simeq \frac{\pi^{2} \kappa^{5 / 2}}{6 N M^{2}}=\frac{81 \pi^{2} M^{3}}{2 N \Lambda^{5}}
$$

Отметим, что полное значение космологической константы, включая плотность вакуумной энергии гравитации и материи, обращается в нуль во всех порядках теории возмущений по степеням параметра $\kappa$ :

$$
\begin{aligned}
\Lambda_{\text {cosmo }} \equiv & \frac{2 \Lambda G_{N}}{\pi^{2}} \int_{-\infty}^{+\infty} d z e^{-4 \rho(z)}\left\{\frac{2 N M^{2}}{3 \kappa}\left(\lambda-6\left[\rho^{\prime}(z)\right]^{2}\right)+\right. \\
& +N \Phi^{\prime 2}(z)+N_{\mathrm{c}} H^{\prime 2}(z)-2 N \Delta_{1} \Phi^{2}(z)-2 N_{\mathrm{c}} \Delta_{2} H^{2}(z)+ \\
& +N \Phi^{4}(z)+N_{\mathrm{c}} \Phi^{2}(z) H^{2}(z)+N_{\mathrm{c}} H^{4}(z)+ \\
& \left.+\frac{2}{3}\left[N \Phi^{2}(z)+N_{\mathrm{c}} H^{2}(z)\right]\left[2 \rho^{\prime \prime}(z)-5 \rho^{\prime 2}(z)\right]\right\}=0,
\end{aligned}
$$

где мы использовали уравнения (25) и (26) для скалярных полей и уравнение (28) для конформного фактора. Для точного сокращения между гравитационным и скалярными вкладами материи для пятимерной космологической константы необходимо также привлечь уравнение (29). Отметим, что включение высших порядков гравитационного взаимодействия, содержащихся в последней строке уравнения (21), не меняет нулевого значения для индуцированной космологической константы $\Lambda_{\text {cosmo }}$ (см. работу [13]).

Получим соотношения между кривизной пространства $A d S, k \simeq 2 \kappa M / 3$, массой Планка $M_{\mathrm{P}}$ и масштабом спонтанного нарушения трансляционной симметрии $M$ :

$$
k^{5} M_{\mathrm{P}}^{4}=\frac{128 N^{2}}{27 \pi^{4}} M^{9}
$$

в соответствии с уравнениями $(27)$ и $(35)$.

Можно также связать пятимерный масштаб Планка с масштабом Планка нашей Вселенной, используя кривизну пространства $A d S_{5}$ :

$$
M_{*}^{3}=\frac{k M_{\mathrm{P}}^{2}}{8 \pi} .
$$

Это приводит к оценке для нижней границы пятимерной массы Планка: $M_{*}>$ $10^{8}$ ГэВ, исходя из экспериментальной оценки для кривизны $k>10 \mathrm{mм}^{-1}=2 \cdot 10^{-3}$ эВ. Последняя основана на отсутствии наблюдаемых отклонений от закона Ньютона на расстояниях более 0.1 мм [17]. Из формулы (46) и упомянутой экспериментальной оценки можно также найти нижние границы для других масштабов модели:

$$
M \geqslant 100 \text { ГэВ, } \quad \Lambda \geqslant 10^{9} \text { ГэВ. }
$$


В том случае, когда эти масштабы достигают минимальных значений, параметры, характеризующие взаимодействия, оказываются весьма малыми:

$$
\zeta \sim \frac{M}{\Lambda} \sim 10^{-7}, \quad \kappa \sim 10^{-13},
$$

поэтому прямое взаимодействие легких частиц сильно подавлено и остается только взаимодействие частиц с гравитацией. Очевидно, что граница для $M \sim 100$ ГэВ является недопустимо низкой, она исключается экспериментами на современных коллайдерах, так как определяет энергетический барьер, выше которого фермионы могут беспрепятственно рапространяться в пятом измерении. В частности, при энергиях, доступных на Тэватроне, гравитация (либо калибровочные бозоны) могла бы придать фермионам достаточно энергии, чтобы исчезнуть из нашей Вселенной.

Для энергий порядка $M \sim 1 \mathrm{T \ni В,} \mathrm{предельных} \mathrm{для} \mathrm{современных} \mathrm{ускорителей,}$ можно оценить масштаб кривизны $A d S_{5}$-пространства $k \sim 10^{-10}$ ГэВ, который соответствует расстояниям порядка $\mu \mathrm{m}$. Такие расстояния недостижимы в экспериментах ближайшего будущего по поиску отклонений от закона Ньютона [17].

В этих пределах скалярные частицы существенно отщепляются от фермионного мира и друг от друга, поскольку

$$
M_{*} \sim 10^{9} \text { ГэВ, } \quad \zeta \sim \frac{M}{\Lambda} \sim 10^{-6.5}, \quad \kappa \sim 10^{-12} .
$$

Частицы типа Хиггса могут быть вовлечены во взаимодействие с калибровочными бозонами и, таким образом, быть наблюдаемыми благодаря испусканию и распаду калибровочных бозонов, а браноны, т.е. кванты поля $\phi$, представляют собой возбуждения геометрической природы, которые можно сопоставить голдстоуновским бозонам вследствие нарушения трансляционной инвариантности. Таким образом, их отщепление от всех других видов материи, кроме гравитации, делает их естественными кандидатами для объяснения состава темной материи/энергии в зависимости от их массы.

Если кривизна пространства $A d S_{5}$ связана с масштабом нарушения электрослабых взаимодействий $k \sim 200$ ГэВ, то граница $M \sim 10^{10}$ ГэВ является слишком высокой, чтобы наблюдать какую-либо реальную физику бранонов в земных лабораториях. Поскольку $\Lambda \sim 10^{14} \Gamma э \mathrm{~B}, \zeta \sim M / \Lambda \sim 10^{-4}$, то в этом случае браноны довольно слабо взаимодействуют и все еще принадлежат темной Вселенной.

Таким образом, для индуцированной гравитации параметр $\zeta$ может стать не слишком малым лишь для $k \sim M \sim \Lambda$ порядка планковских масс, когда гравитация в действительности становится сильной $(\kappa \sim 1)$ и требуется привлечение теории квантовой гравитации.

\section{6. ЗАКЛЮЧЕНИЕ}

В настоящей работе мы обсудили механизм того, как Вселенная может возникнуть на доме́нной стенке (бране) в результате спонтанного нарушения трансляционной симметрии. Оно взаимосвязано с нарушением $\tau$-симметрии в модели пятимерных фермионов с самодействием за счет радиационных вкладов высокоэнергетических фермионов. Низкоэнергетическое эффективное действие для фермионов 
и составных скалярных полей, так же как и индуцированное гравитационное взаимодействие, получено интегрированием высокоэнергетических спинорных степеней свободы в однопетлевом приближении.

Оказывается, что за локализацию легкого вещества на бране отвечают как гравитационные, так и скалярные поля. Классические вакуумные конфигурации этих полей типа браны получены в приближении среднего поля (больших $N_{\text {с и }}$ 》 $N_{\text {c) }}$. Вместе с тем роль гравитации в формировании масс и констант взаимодействия легких частиц незначительна.

Мы исследовали возможный диапазон различных масштабов и констант связи, отталкиваясь от наблюдаемого значения ньютоновской константы, а также используя экспериментальные ограничения на кривизну пространства $A d S_{5}$ и на пороги рождения (исчезновения) высокоэнергетических частиц в экспериментах на современных ускорителях. В результате мы пришли к заключению, что индуцированная гравитация приводит к отщеплению бранонов от вещества Стандартной модели в достаточно широкой области допустимых масштабов и констант связи, делая их, таким образом, естественными кандидатами для объяснения феномена темной материи (или энергии).

Характеризующий силу гравитационного взаимодействия безразмерный параметр оказывается очень малым, порядка $\kappa \leqslant 10^{-8}$. Это обстоятельство оправдывает использование теории возмущений как для вычисления вакуумных конфигураций полей материи и фонового гравитационного поля, так и при выводе спектра масс локализованных частиц.

Благодарности. Эта работа поддержана грантом INFN/IS-PI13. Работа А. А. Андрианова и В. А. Андрианова была частично поддержана РФФИ (грант № 05-02-17477), Программой поддержки ведущих научных школ (грант № НШ5538.2006.2) и Программой "Развитие научного потенциала Высшей школы", подраздел 2.2.1.

\section{Список литературы}

[1] Я. Б. Зельдович, Писвма в ЖКЭТФ, 6 (1967), 883; А. Д. Сахаров, ДАН СССР, 177 (1967), 70.

[2] Д. В. Василевич, Ю. В. Новожилов, ТМФ, 73 (1987), 308; D. V. Vassilevich, Yu. V. Novozhilov, Phys. Lett. B, 220 (1989), 36; Lett. Math. Phys., 21 (1991), 253.

[3] A. A. Andrianov, Yu. V. Novozhilov, Phys. Lett. B, 153 (1985), 422; A. A. Andrianov, Phys. Lett. B, 157 (1985), 425; A. A. Andrianov, V. A. Andrianov, V. Yu. Novozhilov, Yu. V. Novozhilov, Lett. Math. Phys., 11 (1986), 217; Phys. Lett. B, 186 (1987), 401; А. А. Андрианов, В. А. Андрианов, В. Ю. Новожилов, Ю. В. Новожилов, Писвма в ЖКЭТФ, 43 (1986), 8; ТМФ, 70 (1987), 63.

[4] V. A. Rubakov, M. E. Shaposhnikov, Phys. Lett. B, 125 (1983), 136; 139.

[5] K. Akama, "An early proposal of "Brane World"", Gauge Theory and Gravitation, Proc. of Int. Symp. on Gauge Theory and Gravitation (g\&G) (Nara, Japan, 20-24 August, 1982), Lect. Notes Phys., 176, eds. K. Kikkawa, N. Nakanishi, H. Nariai, Springer, Berlin, 1983, 267; hep-th/0001113; M. Visser, Phys. Lett. B, 159 (1985), 22; hep-th/9910093; N. Arkani-Hamed, S. Dimopoulos, G. R. Dvali, Phys. Lett. B, 429 (1998), 263; I. Antoniadis, N. Arkani-Hamed, S. Dimopoulos, G. R. Dvali, Phys. Lett. B, 436 
(1998), 257; M. Gogberashvili, Mod. Phys. Lett. A, 14 (1999), 2025; L. Randall, R. Sundrum, Phys. Rev. Lett., 83 (1999), 3370; 4690.

[6] В. А. Рубаков, УФН, 171 (2001), 913; hep-ph/0104152; УФН, 173 (2003), 219.

[7] I. Antoniadis, "Physics with large extra dimensions", Proc. 2001 European School of HighEnergy Physics (Beatenberg, Switzerland, 26 August - 8 September 2001), eds. N. Ellis, J. March-Russell, CERN, Geneva, 2002, 301 (CERN Yellow Reports for 2002); hep-th/0102202; S. Forste, Fortschr. Phys., 50 (2002), 221.

[8] Yu. A. Kubyshin, Models with extra dimensions and their phenomenology, hep-ph/0111027; J. Hewett, M. Spiropulu, Annual Rev. Nucl. Part. Sci., 52 (2002), 397; R. Dick, Class. Q. Grav., 18 (2001), R1; D. Langlois, Progr. Theor. Phys. Suppl., 148 (2002), 181; R. Maartens, Living Rev. Relativ., 7 (2004), 7; P. Brax, C. van de Bruck, A. C. Davis, Rep. Progr. Phys., 67 (2004), 2183; C. P. Burgess, Ann. Phys., 313 (2004), 283; F. Feruglio, Eur. Phys. J. C, 33 (2004), S114; C. Csaki, TASI Lectures on extra dimensions and branes, hep-ph/0404096.

[9] O. DeWolfe, D. Z. Freedman, S. S. Gubser, A. Karch, Phys. Rev. D, 62 (2000), 046008; M. Gremm, Phys. Lett. B, 478 (2000), 434; Phys. Rev. D, 62 (2000), 044017; C. Csaki, J. Erlich, T. J. Hollowood, Y. Shirman, Nucl. Phys. B, 581 (2000), 309; S. Randjbar-Daemi, M. E. Shaposhnikov, Phys. Lett. B, 492 (2000), 361; G. R. Dvali, M. A. Shifman, Phys. Lett. B, 396 (1997), 64; G. R. Dvali, G. Gabadadze, M. A. Shifman, Phys. Lett. B, 497 (2001), 271.

[10] S. L. Dubovsky, V. A. Rubakov, P. G. Tinyakov, Phys. Rev. D, 62 (2000), 105011; S. L. Dubovsky, V. A. Rubakov, Int. J. Mod. Phys. A, 16 (2001), 4331.

[11] T. Gherghetta, M. E. Shaposhnikov, Phys. Rev. Lett., 85 (2000), 240; M. Laine, H. B. Meyer, K. Rummukainen, M. Shaposhnikov, JHEP, 0301 (2003), 068; 0404 (2004), 027.

[12] A. A. Andrianov, V. A. Andrianov, P. Giacconi, R. Soldati, JHEP, 0207 (2003), 063.

[13] A. A. Andrianov, V. A. Andrianov, P. Giacconi, R. Soldati, JHEP, 0507 (2005), 003.

[14] Р. Раджараман, Солитоны и инстантоны в квантовой теории поля, Мир, М., 1985; A. Vilenkin, Phys. Rep., 121 (1985), 263; R. MacKenzie, Nucl. Phys. B, 303 (1988), 149; Г. Е. Воловик, Писъма в ЖЭЭФ, 75 (2002), 63; D. Bazeia, R. F. Ribeiro, M. M. Santos, Phys. Rev. D, 54 (1996), 1852.

[15] A. Dobado, A. L. Maroto, Nucl. Phys. B, 592 (2001), 203; J. Alcaraz, J. A. R. Cembranos, A. Dobado, A. L. Maroto, Phys. Rev. D, 67 (2003), 075010; J. A. R. Cembranos, A. Dobado, A. L. Maroto, Phys. Rev. D, 68 (2003), 103505; 70 (2004), 096001.

[16] A. A. Andrianov, L. Bonora, Nucl. Phys. B, 233 (1984), 232; 247.

[17] E. G. Adelberger, B. R. Heckel, A. E. Nelson, Annual Rev. Nucl. Part. Sci., 53 (2003), 77.

[18] G. Cognola, P. Giacconi, Phys. Rev. D, 39 (1989), 2987.

[19] D. Vassilevich, Phys. Rep., 388 (2003), 279.

[20] W. Pauli, Teoria della Relatività, Boringhieri, Torino, 1970; В. А. Фок, Теория пространства, времени и тяготения, ГИТТЛ, М., 1955.

[21] V. A. Miransky, M. Tanabashi, K. Yamawaki, Phys. Lett. B, 221 (1989), 177; W. A. Bardeen, C. T. Hill, M. Lindner, Phys. Rev. D, 41 (1990), 1647. 